\title{
Constitutive equations with pressure-dependent rheological parameters for describing ice creep
}

\author{
MARTINA ARCANGIOLI, ${ }^{1}$ ANGIOLO FARINA, ${ }^{1}$ LORENZO FUSI, ${ }^{1}$ \\ GIUSEPPE SACCOMANDI ${ }^{2}$
}

\author{
${ }^{1}$ Dipartimento di Matematica e Informatica 'U. Dini', Università degli Studi di Firenze, Viale Morgagni 67/a, 50134 Firenze, \\ Italy \\ ${ }^{2}$ Dipartimento di Ingegneria, Università di Perugia, Via G. Duranti 93, 06125 Perugia, Italy \\ Correspondence: Angiolo Farina <angiolo.farina@unifi.it>
}

\begin{abstract}
Experimental data from creep tests on polycrystalline ice samples highlight not only the non-Newtonian behavior of ice but also suggest a critical dependence of the various rheological parameters upon the applied hydrostatic pressure. We propose a new modeling framework, based on implicit theories of continuum mechanics, that generalizes two well-known constitutive models by taking into account the effect of the pressure in the description of ice in creep. To ascertain the validity of the proposed models, we fit the physical parameters with experimental data for the elongational flow of ice samples. The results show good agreement with the experimental creep curves. In particular, the proposed generalized models reproduce the increase of the creep rate due to the presence of hydrostatic pressure.
\end{abstract}

KEYWORDS: glacier flow, ice dynamics, ice rheology

\section{INTRODUCTION}

An understanding of the physical processes that take place within glaciers requires information about the plastic deformation behavior of ice at high pressures. Jones and Chew (1983) and Mctigue and others (1985) reported creep experiments on polycrystalline ice subject to a combination of uniaxial compression and hydrostatic pressure. Further, Barrette and Jordaan (2003) investigated the compressive behavior of laboratory-produced ice as well as genuine iceberg ice subjected to constant confinement pressure.

Passman (1982) obtained an exact solution for the deformation in biaxial creep tests using the constitutive equation of a second-grade fluid. In his model, there are three constant constitutive parameters (including the viscosity). Mctigue and others (1985) showed that these constitutive parameters exhibit a non-negligible dependence on the applied hydrostatic pressure (the confining pressure). They ascribed this phenomenon to a partial inadequacy of the second-grade fluid model. They stated 'The results presented here, then, are not intended to suggest that the second-order fluid model gives a complete representation for the behavior of ice.'

The fact that the ice parameters (obtained by fitting the experimental data) show significantly different values at different pressures were also emphasized by Jones and Chew (1983), who wrote 'It has always been assumed that the application of hydrostatic pressure increases the creep rate of ice.'This effect was noticed for the first time by Rigsby (1958), who stated: 'the shear strain rate increased as the pressure was increased at constant temperature, but that rate is practically independent of hydrostatic pressure when the difference between the ice temperature and the melting temperature is kept constant.'

Barrette and Jordaan (2003) reported a number of experimental tests, designed to investigate the deformation of ice under a constant compressive load and at various confinement pressures. In particular, the testing conditions (confinements and axial stress) are thought to be representative of those that can occur in interactions between icebergs and engineered structures, such as the hull of a ship. Despite such experimental evidence, few theoretical explanations have been proposed so far; see Glen (1955), Van Der Veen (2013), Section 6.2 of Schulson and Duval (2009); Greve and others (2014).

Recently, Rajagopal (2003, 2006) argued that it is often advantageous to write constitutive relations in implicit forms. In this perspective, the material parameters characterizing the constitutive model can depend on the stress itself and therefore also on the pressure (the latter being the trace of the Cauchy stress tensor, Rajagopal (2015)).

Our purpose is to propose two models that can explain the experimental results concerning primary creep (or transient creep) and secondary creep (Jones and Chew (1983); Mctigue and others (1985); Barrette and Jordaan (2003)), which include a rheology depending significantly upon the pressure. This fact can be observed in Figure 3 of Barrette and Jordaan (2003) or in Figure 2 of Mctigue and others (1985), where the effect of the pressure on the rheological behavior of ice becomes evident. Thus, starting from the second-grade fluid model, we consider two constitutive equations in which we add the fundamental hypothesis that the rheological parameters depend on the pressure. We select the second-grade fluid model because it is known that this model can represent primary and secondary (but not tertiary) creep (Passman (1982)), while a power-law model can represent only secondary creep.

The proposed models are based on (i) an equation which generalizes the classical second grade constitutive equation (Mctigue and others (1985)), and (ii) an equation which is a generalization of a power-law fluid of grade 2 (Man and Sun (1987)). Model (i) is a generalization of the simplest fluid model of differential type that can capture normal 
stress differences. However, since second-grade models usually fail to represent the non-linear rate dependence of ice in shear (Mctigue and others (1985); Man and Sun (1987)) we also consider model (ii) that accounts for both shear thinning (as in Glen's law) and normal stress differences.

We believe that (i) and (ii) are improvements on the models of Mctigue and others (1985) and Man and Sun (1987) because, given the high pressures that develop within glaciers, we expect the viscosity to depend also on the pressure (as experimentally highlighted by Jones and Chew (1983), Mctigue and others (1985) and Barrette and Jordaan (2003)). The dependence of the viscosity on the pressure is indeed a well-known phenomenon (see, for instance, Bridgman (1958) for a discussion of the early experimental work concerning the dependence of viscosity on pressure). Further, models (i) and (ii) can generate normal stress effects. This phenomenon was highlighted by Mctigue and others (1985) and Man and Sun (1987), where the freesurface depression of a second-grade fluid flowing down an inclined open semi-circular channel is estimated.

In this paper, we analyze the one-dimensional (1-D) flow experienced by ice samples during a creep test, assuming that the rheological parameters follow a Barus-type law ${ }^{1}$ (Barus (1893); Bridgman (1958); Szeri (2005)). The specific choice of the Barus law is mainly guided by empiricism and the requirement of simplicity. We present a similar analysis for both models (i) and (ii), showing how the dependence of the rheological parameters on pressure has nonnegligible qualitative and quantitative effects on the flow. Actually, both models describe quite well the experimental results presented in Jones and Chew (1983), Mctigue and others (1985) and Barrette and Jordaan (2003), and capture the increase in the creep rate caused by the increasing pressure.

The paper is organized as follows: in the first section, we present models (i) and (ii). In the second section, the governing equations for creep flow are derived. In the third section, we illustrate the fitting of the parameters against the experimental data relating to primary and secondary creep. The last section is devoted to some concluding remarks.

\section{THE MODEL}

We consider a class of constitutive equations originating from the second-grade fluid model of implicit type (Rajagopal (2003, 2006); Rajagopal and Szeri (2003)) of the form

$$
f\left(\mathbf{T}, \mathbf{A}_{1}, \mathbf{A}_{2}, \ldots, \mathbf{A}_{n}\right)=0,
$$

where $\mathbf{T}$ is the Cauchy stress tensor and $\mathbf{A}_{n}$ are the RivlinEricksen tensors (Passman (1982); Truesdell and Rajagopal (2000))

$$
\begin{gathered}
\mathbf{A}_{1}=\left(\nabla \mathbf{v}+\nabla \mathbf{v}^{\top}\right)=\mathbf{G}+\mathbf{G}^{\top}, \\
\mathbf{A}_{2}=\frac{\mathrm{d} \mathbf{A}_{1}}{\mathrm{~d} t}+\mathbf{A}_{1} \mathbf{G}+\mathbf{G}^{\top} \mathbf{A}_{1}, \\
\mathbf{A}_{n}=\frac{\mathrm{d} \mathbf{A}_{n-1}}{\mathrm{~d} t}+\mathbf{A}_{n-1} \mathbf{G}+\mathbf{G}^{\top} \mathbf{A}_{n-1} .
\end{gathered}
$$

1 The viscosity $\eta$ of many fluids varies roughly exponentially with the pressure $p$, as found by Barus (1893), namely $\eta=$ $\eta_{0} e^{k_{n} p}$ where $\eta_{0}$ is the viscosity at a specific reference pressure.
The tensor $\mathbf{G}$ in Eqns (2)-(4) denotes the gradient of the velocity field $\boldsymbol{v}(\boldsymbol{x}, t)$, i.e. $\nabla \boldsymbol{v}=\mathbf{G}$. In particular, $\mathbf{G}=\dot{\mathbf{F}} \mathbf{F}^{-1}$ where $\mathbf{F}$ is the deformation gradient tensor.

We assume mechanical incompressibility so that

$$
\operatorname{tr} \mathbf{A}_{1}=0
$$

\subsection{Second-grade fluid type constitutive models}

We select second-grade fluids (Truesdell and Rajagopal (2000)) because Mctigue and others (1985) show that these models give an excellent representation of primary creep of ice at constant confining pressure. More importantly, Truesdell and Noll (1992) show that second-grade fluids exhibit normal stress differences, a feature which powerlaw constitutive models cannot capture and which seems to play a significant role in ice deformation.

As the first constitutive model, we generalize the basic second-grade fluid model proposed in Mctigue and others (1985), assuming that the characteristic constitutive parameters depend on the pressure $p$, the mean normal stress (Rajagopal and others (2012, 2015); Rajagopal (2015); Rajagopal and Saccomandi (2016)):

$$
p=-\frac{1}{3} \operatorname{tr} \mathbf{T}
$$

We thus consider ${ }^{2}$

$$
\begin{aligned}
\mathbf{T}= & -p \mathbf{I}+\mu(p) \mathbf{A}_{1}+\alpha_{1}(p)\left[\mathbf{A}_{2}-\frac{1}{3}\left(\operatorname{tr} \mathbf{A}_{2}\right) \mathbf{I}\right]+\alpha_{2}(p) \\
& \times\left[\mathbf{A}_{1}^{2}-\frac{1}{3}\left(\operatorname{tr} \mathbf{A}_{1}^{2}\right) \mathbf{I}\right]
\end{aligned}
$$

where $\operatorname{tr}(\mathbf{T}+p \mathbf{I})=0$. As stated earlier, we assume that the coefficients characterizing the constitutive Eqn (6) of the fluid may depend on $p$ so that the constitutive model is implicit. Such an assumption, as we shall see, plays a key role in the analysis of the flow.

Next, we impose the following restrictions on the constitutive parameters:

$$
\mu \geq 0, \quad \alpha_{1}<0, \quad \alpha_{1}+\alpha_{2} \neq 0,
$$

which are suggested by some experiments performed on polymer solutions and melts (Bird and others (1977)). Using thermodynamic arguments, Dunn and Fosdick (1974) showed that $\alpha_{1} \geq 0$ and $\alpha_{2}+\alpha_{1}=0$. In particular, if $\alpha_{1}$ is positive the Clausius-Duhem inequality is met and the Helmholtz potential is a minimum when the fluid is at rest. Fosdick and Rajagopal (1979) showed that, irrespectively of the sign of $\alpha_{1}+\alpha_{2}$, the fluid model exhibits unacceptable stability characteristics when $\alpha_{1}$ is negative. We refer to Dunn and Rajagopal (1995) for a detailed discussion on the relevant issues concerning second-grade fluids. These restrictions, however, need not be straightforwardly applicable to our models, since we are considering implicit constitutive equations. Indeed, referring to Fosdick and Rajagopal (1979), we remark that their results are obtained by using a relation involving an $L^{2}$ estimate of $\boldsymbol{v},\left\|\mathbf{A}_{1}\right\|$ and an $L^{1}$ estimate

2 The dimensions of the coefficients are

$$
[\mu]=\frac{\text { mass }}{l \cdot \text { time }^{2}},\left[\alpha_{1}\right]=\left[\alpha_{2}\right]=\frac{\text { mass }}{l} .
$$


of $\left\|\mathbf{A}_{1}\right\|^{3}$, in which the material moduli $\mu, \alpha_{1}$ and $\alpha_{2}$ are taken constant. Such an approach is no longer applicable in our case since the material moduli depend on the stress itself. In other words, the separation between kinematic variables and stress (on which the procedure developed in Fosdick and Rajagopal (1979) is based) cannot be performed in our case. Therefore, following Mctigue and others (1985), assumptions (7) are essentially based on experimental evidence.

As a second constitutive model, we consider a generalized version of the one proposed by Man and Sun (1987):

$$
\begin{aligned}
\mathbf{T}= & -p \mathbf{I}+\mu(p)\left[a_{0}+\frac{1}{2}\left(\operatorname{tr} \mathbf{A}_{1}^{2}\right)\right]^{m / 2} \mathbf{A}_{1}+\alpha_{1}(p) \\
& \times\left[\mathbf{A}_{2}-\frac{1}{3}\left(\operatorname{tr} \mathbf{A}_{2}\right) \mathbf{I}\right]+\alpha_{2}(p)\left[\mathbf{A}_{1}^{2}-\frac{1}{3}\left(\operatorname{tr} \mathbf{A}_{1}^{2}\right) \mathbf{I}\right],
\end{aligned}
$$

where $a_{0}$ and $m$ are constants which depend on the nature of the ice. Following Kannan and Rajagopal (2013), we consider $m=-(2 / 3)$ and $a_{0}=0$, but here we assume that the restrictions (7) hold true.

We consider the constitutive Eqn (8) because it is a simple model exhibiting both normal stress differences and shear thinning effects, which agrees with the power law proposed by Glen (1955). In fact, some experimental results clearly indicate that glacier ice shows shear thinning (Kjartanson and others (1988)). In a sense, our approach is parallel to the one presented by Man and Sun (1987), where the creep data of Mctigue and others (1985) were used to determine the material parameters.

Before proceeding further, we emphasize that, in general, the second-grade fluid model gives rise to considerable mathematical issues. Indeed, the related mathematical problems involve derivatives of third order, i.e. one order higher than in the Navier-Stokes equation. Therefore, when considering fully 3-D flows, one needs additional boundary conditions (Rajagopal and Kaloni (1989); Rajagopal (1995)). However, this problem can be circumvented when the constitutive model is written in an implicit form. Indeed, the constitutive relation is solved together with the balance of linear momentum, without substituting the expression for the stress into the balance of linear momentum. So the order of the differential equations is not increased and no further boundary conditions are needed. Clearly, such a procedure works also when the material coefficients are constant. We refer the readers to Rajagopal (2003) where this issue is discussed at length.

Concerning the dependence on $p$ of the parameters $\mu$ and $\alpha_{2}$, we consider a Barus-type law (Barus (1893); Bridgman (1958)), while $\alpha_{1}$ is assumed not to depend on $p$ :

$$
\begin{aligned}
\mu(p) & =\mu_{0} \exp \left(-k_{\mu} \frac{p-p_{\text {ref }}}{p_{\text {ref }}}\right), \\
\alpha_{2}(p) & =\alpha_{2,0} \exp \left(-k_{\alpha} \frac{p-p_{\text {ref }}}{p_{\text {ref }}}\right),
\end{aligned}
$$

$\alpha_{1}=$ constant with respect to the pressure $p$,

with $\mu_{0}, \alpha_{2,0}, k_{\mu}$ and $k_{\alpha}$ constants and where $p_{\text {ref }}$ is a reference pressure.

For (9), we selected a Barus-type law simply because it gives a good fit in many contexts. For instance, we find an exponential variation in the viscosity due to pressure in problems such as elastohydrodynamics (Szeri (2005)), in problems involving the flow of geological matter (Sahaphol and Miura (2005)), and the response of composite materials (Shin and Pae (1992)). The properties of polymeric materials may also vary exponentially with pressure (Jones Parry and Tabor (1973)). We, however, remark that, to our knowledge, there are no experiments showing that the ice viscosity varies exponentially with pressure.

The issue of pressure-dependent viscosity is also analyzed by Kannan and Rajagopal (2013), who propose a model that takes into account both the pressure and the volume fraction of the rock sands and grains trapped within the interstices of the rock glacier. Our context is different: we consider experiments performed on a sample of polycrystalline ice with no impurities trapped inside.

Assumption (10) is new since there are no theoretical or experimental studies suggesting it. The choice of this law is inspired by the rheology of some organic liquids.

Finally, we assume (11) following Kannan and Rajagopal (2013). Actually, in fitting the experimental data, we found that $\alpha_{1}$ is practically insensitive to the pressure variations (at least in the range of the considered data).

\section{CREEP FLOW}

We consider a sample of polycrystalline ice and select a Cartesian coordinate system. The sample has length $I_{0}$ in the undeformed reference configuration (see Fig. 1a). We then consider an isochoric homogeneous flow (elongational or compression flow) as in creep flow tests (see Fig. 1b), where $I(t)$ denotes the sample length at time $t$, and

$$
\beta(t)=\frac{l(t)}{I_{0}}
$$

is the stretch. Obviously $\beta(t)$ is related to the axial strain $\epsilon(t)=$ $\left(I(t)-I_{0}\right) / I_{0}$ by $\beta(t)=\epsilon(t)+1$. We also introduce the strain rate $^{3} \dot{\epsilon}=\dot{\beta}$, i.e. the change in deformation with respect to time.

Proceeding as in Passman (1982) and Mctigue and others (1985), the deformation gradient tensor is diagonal and has the form ${ }^{4}$

$$
\mathbf{F}=\operatorname{diag}\left[\beta(t), \frac{1}{\sqrt{\beta(t)}}, \frac{1}{\sqrt{\beta(t)}}\right]
$$

while the Rivlin-Ericksen tensors $\mathbf{A}_{1}, \mathbf{A}_{2}$ take the diagonal forms

$$
\begin{gathered}
\mathbf{A}_{1}=\operatorname{diag}[2 a,-a,-a], \\
\mathbf{A}_{2}=\operatorname{diag}\left[2 \dot{a}+4 a^{2},-\dot{a}+a^{2},-\dot{a}+a^{2}\right],
\end{gathered}
$$

where

$$
a(t)=\frac{\dot{\beta}(t)}{\beta(t)} .
$$

For the Cauchy stress tensor, we have

$$
\mathbf{T}=\operatorname{diag}\left[f_{A}(t), f_{L}(t), f_{L}(t)\right],
$$

3 The superposed dot indicates differentiation respect to time. 4 It easy to check that the isochoricity condition is fulfilled, namely $\operatorname{det} \mathbf{F}=1$. 


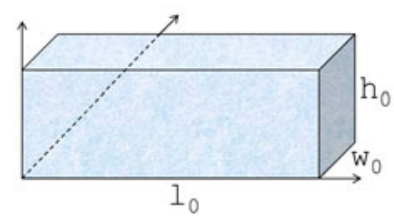

b

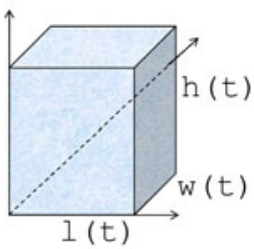

Fig. 1. Reference and current configuration.

where $f_{A}(t)$ is the stress along the $x$-axis (axial stress) and $f_{L}(t)$ is the lateral stress, also referred to as confining pressure (Jones and Chew (1983); Mctigue and others (1985)). The loads are portrayed in Fig. 2.

Applying the stress equilibrium equation and the model (6), we obtain the differential equation

$$
f_{A}-f_{L}=3 a \mu(p)+3 \alpha_{1} \dot{a}+3 a^{2}\left(\alpha_{1}+\alpha_{2}(p)\right),
$$

with the initial condition $a(0)=a_{0}$.

The pressure is given by

$$
p=-\frac{1}{3}\left[f_{A}+2 f_{L}\right]
$$

Following Passman (1982) and assuming that $f_{A}, f_{L}$ are constant in time, Eqn (15) can be solved analytically. To see this, we introduce the following parameters

$$
\begin{gathered}
\nu(p)=\frac{\mu(p)}{2\left(\alpha_{1}+\alpha_{2}(p)\right)}, \\
\chi(p)=1+\frac{\alpha_{2}(p)}{\alpha_{1}}, \\
b^{2}(p)=\frac{\mu^{2}(p)}{4\left(\alpha_{1}+\alpha_{2}(p)\right)^{2}}+\frac{f_{A}-f_{L}}{3\left(\alpha_{1}+\alpha_{2}(p)\right)},
\end{gathered}
$$

which, unlike in Passman (1982), depend on $p$ because of (9) and (10).

Equation (15) coincides with equation (29) of Passman (1982). We can solve it analytically because both $f_{A}$ and $f_{L}$ are constant in time. The solution procedure is equal to the one illustrated by Passman (1982). Indeed, since $p$ does not change in time, the parameters in (15) are constant. Therefore we keep the main result of Passman (1982), limiting our discussion to the case $b^{2}>0$. The latter yields

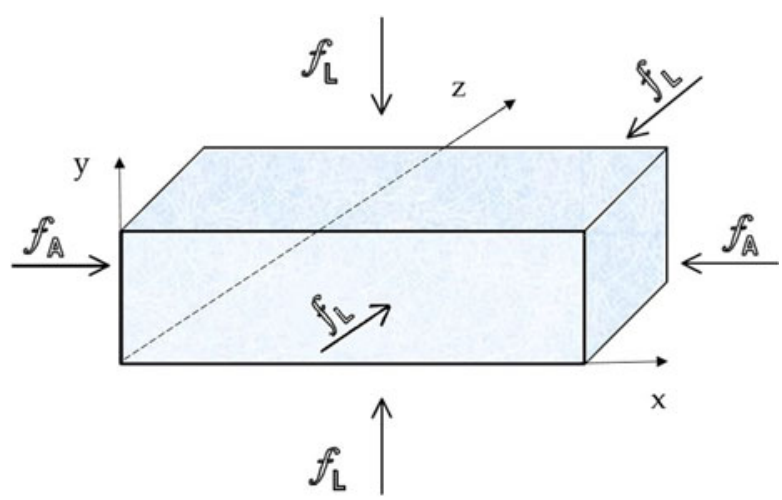

Fig. 2. A sample subjected to a compressive force $f_{A}$ and a confining pressure $f_{L}$.

physically reasonable results and provides the best fits to laboratory creep data, as shown by Passman (1982) and Mctigue and others (1985). We will check a posteriori that the assumption $b^{2}>0$ is actually fulfilled, at least in the considered range of pressures. Of course, outside this range, it could happen that $b^{2}$ becomes negative, simply because

$$
f_{A}-f_{L}<-\frac{3 \mu^{2}(p)}{4\left(\alpha_{1}+\alpha_{2}(p)\right)} \text {. }
$$

Should this occur, then there would be a critical time at which the deformation rate and the strain become undefined. This fact does not mean that the model fails, but that homogeneous deformations do not exist anymore.

So, considering $b^{2}>0$, we obtain

$$
\frac{a(t)+v}{b}=\frac{\left[\left(a_{0}+v\right) / b\right] \cosh (\chi b t)+\sinh (\chi b t)}{\left[\left(a_{0}+v\right) / b\right] \sinh (\chi l t)+\cosh (\chi b t)},
$$

and

$$
\epsilon(t)=\beta_{0}\left[\cosh (\chi b t)+\left(\frac{a_{0}+v}{b}\right) \sinh (\chi b t)\right]^{\frac{1}{\chi}} \mathrm{e}^{-v t}-1
$$

$\beta_{0}$ being the initial stretch.

If instead of the constitutive model (6) we consider (8) with $m=-2 / 3$ and $a_{o}=0$, we obtain the differential equation:

$$
\dot{\mathrm{a}}=-\lambda(p) \mathrm{a}^{1 / 3}+\chi(p) \mathrm{a}^{2}+c(p),
$$

where

$$
\lambda(p)=\sqrt[3]{\frac{1}{3}} \frac{\mu(p)}{\alpha_{1}(p)}, \quad c(p)=\frac{f_{A}-f_{L}}{3 \alpha_{1}(p)} .
$$

Equation (19) is solved numerically assuming that $f_{A}$ and $f_{L}$ are constant in time.

\section{FITTING OF THE PARAMETERS WITH EXPERIMENTAL DATA}

In order to validate the models (6) and (8), along with the assumptions (9)-(11), we consider the experimental data reported in Jones and Chew (1983), Mctigue and others (1985) and Barrette and Jordaan (2003), which refer to a series of creep flow tests performed on polycrystalline ice samples, maintained at constant temperature, at different confinement pressures.

First, we focus on the data shown by Mctigue and others (1985) and Jones and Chew (1983) since they are the same experimental data but presented in different forms. Then we analyze the experimental data of Barrette and Jordaan (2003). So, starting with Mctigue and others (1985) and Jones and Chew (1983), we denote by:

1. the data analysis presented in Mctigue and others (1985), 2. the data analysis illustrated in Jones and Chew (1983).

In particular, data analysis 2 consists essentially of the creep curves shown in Figure 1 of Jones and Chew (1983), where the strain rate is plotted against strain under different confining pressures: 0 and $50 \mathrm{MPa}$. The difference between the two curves is obvious, meaning that the rheological properties of ice are different at different pressures, as remarked by Jones and Chew (1983). 
Data analysis 1 refers to Figure 2 of Mctigue and others (1985), which displays the axial strain vs. time corresponding to three different confining pressures: atmospheric pressure, 37 and $50 \mathrm{MPa}$. Also, in this case, the slopes of the curves increase with pressure. The first and last curves differ by a factor $\sim 2$.

The five parameters $\mu_{0}, k_{\mu}, \alpha_{2, \sim 0}, k_{\alpha \prime} \alpha_{1}$ characterizing (18) and the differential Eqn (19) are obtained by fitting to the experimental data. Since the data refer to the same experiments, we have fitted the coefficients by considering analyses 1 and 2 separately. By comparing the results obtained, we are able to evaluate the validity of the proposed models.

The outcomes of the best-fit procedures (whose details are not shown here) are summarized in Tables 1 (data analysis 1 ) and 2 (data analysis 2), where (a) refers to equation (18), i.e. model (6), and (b) refers to equation (19), i.e. model (8).

In Table 1 a we report the values of the coefficients $\mu_{0}, k_{\mu}$ $\alpha_{2, \sim 0}, k_{\alpha \prime} \alpha_{1}$, obtained by fitting the axial strain $\epsilon(t)$ given by (16) to the data analysis 1 , while Table $1 \mathrm{~b}$ shows the same five parameters obtained by fitting $\epsilon(t)$ given by (19).

The coefficients of Tables $1 \mathrm{a}$ and $2 \mathrm{a}$ are similar, with differences being of the order $15-20 \%$. This observation strengthens model (6) since we obtain very similar values for the rheological parameters. By contrast, the coefficients of Tables $1 \mathrm{~b}$ and $2 \mathrm{~b}$ differ more from each other. In particular, we note a significant difference in $k_{\alpha}$.

The fittings are shown graphically in Figs 3-6. Figures 3 and 4 refer to $\epsilon(t)$ given by equation (18), i.e. the constitutive model (6). In Fig. 3 we plot the data analysis illustrated in Mctigue and others (1985) (circles), and the axial strain $\epsilon(t)$ computed with the coefficients of Table 1. Figure 4 shows $\epsilon$ vs. $\dot{\epsilon}$, computed with $\mu_{0}, k_{\mu}, \alpha_{2, \sim 0}, k_{\alpha \prime} \alpha_{1}$ of Table $2 \mathrm{a}$ and the data of Jones and Chew (1983) (circles). Figures 5 and 6

Table 1. Constitutive coefficients and pressures for creep tests, obtained by fitting to the experimental data of Mctigue and others (1985)

(a) is for the constitutive model (6) (see also Fig. 3)

\begin{tabular}{llccc}
\hline$\mu_{0}$ & Pas & $5.9 \times 10^{13}$ & \\
$k_{\mu}$ & & $3.6 \times 10^{-3}$ & \\
$\alpha_{2,0}$ & Pas $^{2}$ & & $5.4 \times 10^{21}$ & \\
$k_{\alpha}$ & & & $7.1 \times 10^{-3}$ & \\
$\alpha_{1}$ & $\mathrm{Pas}^{2}$ & & $-1.8 \times 10^{19}$ & \\
$f_{L}$ & $\mathrm{MPa}$ & 0 & -37 & -50 \\
$f_{A}$ & $\mathrm{MPa}$ & -0.47 & -37.47 & -50.47 \\
$\epsilon_{0}$ & & $-3 \times 10^{-3}$ & $-4 \times 10^{-3}$ & $-5 \times 10^{-3}$ \\
$\beta_{0}$ & & $1-3 \times 10^{-3}$ & $1-4 \times 10^{-3}$ & $1-5 \times 10^{-3}$ \\
$a_{0}$ & $\mathrm{~s}^{-1}$ & $-3.7 \times 10^{-8}$ & $-4.5 \times 10^{-8}$ & $-3.03 \times 10^{-8}$ \\
$p$ & $\mathrm{MPa}$ & 0.16 & 37.16 & 50.16 \\
\hline
\end{tabular}

(b) is for the constitutive model (8) (see also Fig. 5

\begin{tabular}{llccc}
\hline$\mu_{0}$ & Pas & & $0.1 \times 10^{7}$ & \\
$k_{\mu}$ & & & $1.8 \times 10^{-3}$ & \\
$\alpha_{2,0}$ & Pas $^{2}$ & & $8.4 \times 10^{21}$ & \\
$k_{\alpha}$ & & & $2.8 \times 10^{-3}$ & \\
$\alpha_{1}$ & $\mathrm{Pas}^{2}$ & & $-6.1 \times 10^{19}$ & \\
$f_{L}$ & $\mathrm{MPa}$ & 0 & -37 & -50 \\
$f_{A}$ & $\mathrm{MPa}$ & -0.47 & -37.47 & -50.47 \\
$\epsilon_{0}$ & & $-3 \times 10^{-3}$ & $-4 \times 10^{-3}$ & $-5 \times 10^{-3}$ \\
$\beta_{0}$ & & $1-3 \times 10^{-3}$ & $1-4 \times 10^{-3}$ & $1-5 \times 10^{-3}$ \\
$a_{0}$ & $\mathrm{~s}^{-1}$ & $-2.8 \times 10^{-8}$ & $-3 \times 10^{-8}$ & $-2.9 \times 10^{-8}$ \\
$p$ & $\mathrm{MPa}$ & 0.16 & 37.16 & 50.16 \\
\hline
\end{tabular}

Table 2. Constitutive coefficients and pressures for creep tests, obtained by fitting to the experimental data of Jones and Chew (1983)

\begin{tabular}{|c|c|c|c|}
\hline $\begin{array}{l}\mu_{0} \\
k_{m}\end{array}$ & Pas & \multicolumn{2}{|c|}{$\begin{array}{c}5 \times 10^{13} \\
3 \times 10^{-3}\end{array}$} \\
\hline $\begin{array}{l}\kappa_{\mu} \\
\alpha_{2,0}\end{array}$ & $\mathrm{Pas}^{2}$ & \multicolumn{2}{|c|}{$\begin{array}{l}3.1 \times 10 \\
3.8 \times 10^{21}\end{array}$} \\
\hline$k_{\alpha}$ & & \multicolumn{2}{|c|}{$6.8 \times 10^{-3}$} \\
\hline$\alpha_{1}$ & $\mathrm{Pas}^{2}$ & \multicolumn{2}{|c|}{$-2 \times 10^{19}$} \\
\hline$f_{L}$ & $\mathrm{MPa}$ & 0 & -50 \\
\hline$f_{A}$ & $\mathrm{MPa}$ & -0.47 & -50.47 \\
\hline$\epsilon_{0}$ & & $-5 \times 10^{-3}$ & $-4 \times 10^{-3}$ \\
\hline$\beta_{0}$ & & $1-5 \times 10^{-3}$ & $1-4 \times 10^{-3}$ \\
\hline$\alpha_{0}$ & $\mathrm{~s}^{-1}$ & $-1.4 \times 10^{-8}$ & $-3.0 \times 10^{-8}$ \\
\hline$p$ & $\mathrm{MPa}$ & 0.16 & 50.16 \\
\hline
\end{tabular}

(b) is for the constitutive model (8) (see also Fig. 6

\begin{tabular}{|c|c|c|c|}
\hline $\begin{array}{c}\mu_{0} \\
k_{\mu}\end{array}$ & Pas & \multicolumn{2}{|c|}{$\begin{array}{c}2 \times 10^{6} \\
1.9 \times 10^{-3}\end{array}$} \\
\hline$\alpha_{2,0}$ & $\mathrm{Pas}^{2}$ & \multicolumn{2}{|c|}{$8.8 \times 10^{21}$} \\
\hline$k_{\alpha}$ & & \multicolumn{2}{|c|}{$9.3 \times 10^{-3}$} \\
\hline$\alpha_{1}$ & $\mathrm{Pas}^{2}$ & \multicolumn{2}{|c|}{$-3.1 \times 10^{19}$} \\
\hline$f_{L}$ & $\mathrm{MPa}$ & 0 & -50 \\
\hline$f_{A}$ & $\mathrm{MPa}$ & -0.47 & -50.47 \\
\hline$\epsilon_{0}$ & & $-5 \times 10^{-3}$ & $-4 \times 10^{-3}$ \\
\hline$\beta_{0}$ & & $1-5 \times 10^{-3}$ & $1-4 \times 10^{-3}$ \\
\hline$a_{0}$ & $\mathrm{~s}^{-1}$ & $-1.4 \times 10^{-8}$ & $-3.0 \times 10^{-8}$ \\
\hline$p$ & $\mathrm{MPa}$ & 0.16 & 50.16 \\
\hline
\end{tabular}

refer to equation (19) (constitutive model (8)). Figure 5 shows the comparison with the analysis of Mctigue and others (1985), and Fig. 6 displays the data by Jones and Chew (1983) (circles) and $\epsilon, \dot{\epsilon}$, computed using the parameters of Table $2 \mathrm{~b}$.

A second set of experimental data, independent of those reported by Mctigue and others (1985) and Jones and Chew (1983), is shown in Figure 3 of Barrette and Jordaan (2003). These data reveal the onset of tertiary creep for longer times. However, for the purpose of fitting models (6) and (8), the data are truncated at the onset of tertiary creep. The coefficients obtained by fitting the three curves reported in Fig. 3 of Barrette and Jordaan (2003) are listed in Tables 3a

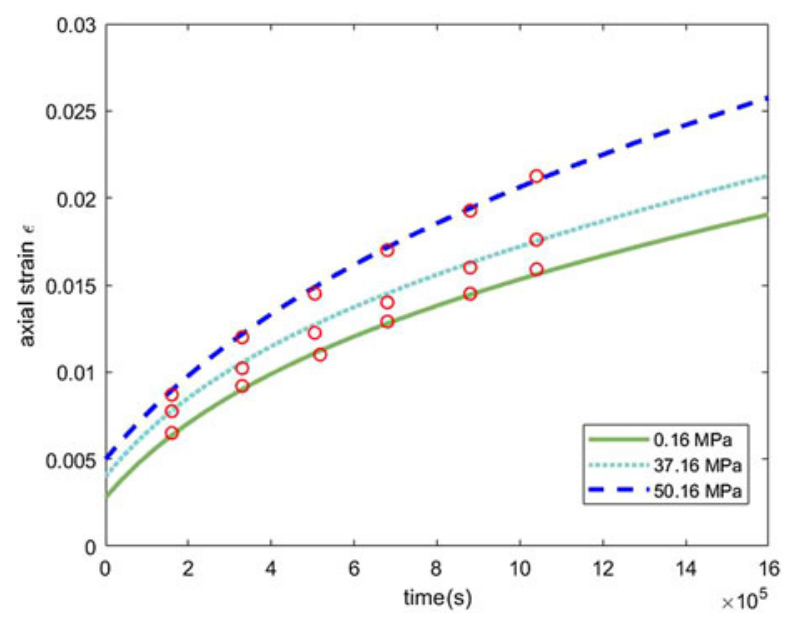

Fig. 3. Axial strain vs. time for different confining pressures, computed with model (6) and the coefficients of Table 1. Experimental data (small circles) of Mctigue and others (1985). 


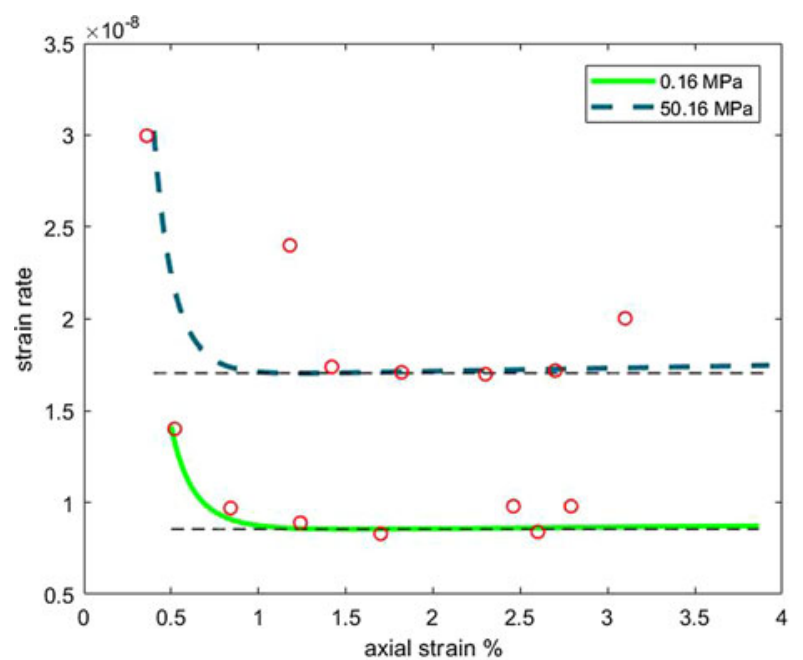

Fig. 4. Strain rate vs. axial strain for different confining pressures, computed with model (6) and the coefficients of Table 2. Experimental data (small circles) of Jones and Chew (1983).

and 3b. Now the axial stress $f_{A}$ is $f_{L}+15 \mathrm{MPa}$, while in Mctigue and others (1985) and Jones and Chew (1983) $f_{A}=f_{L}+0.47 \mathrm{MPa}$. Moreover, the order of magnitude of strain rate is $\sim 10^{5}$ times larger than that of Mctigue and others (1985) and Jones and Chew (1983).

The fitted curves are shown in Figs 7 and 8. In particular, Fig. 7 displays the comparison between the data by Barrette and Jordaan (2003) and the model (6), while Fig. 8 shows the comparison with model (8). Actually, both models reproduce quite well the primary and secondary creep stages.

We finally remark that assumption $b^{2}>0$ is always fulfilled with the data of Tables $1 \mathrm{a}, 2 \mathrm{a}$ and $3 \mathrm{a}$.

\section{RESULTS AND DISCUSSION}

It is well known that ice under applied stress experiences creep deformation. Thus creep tests, performed at constant stress, are often used to determine the deformation of the ice and, consequently, its rheological parameters.

The experimental data reported by Barrette and Jordaan (2003), Mctigue and others (1985) and Jones and Chew

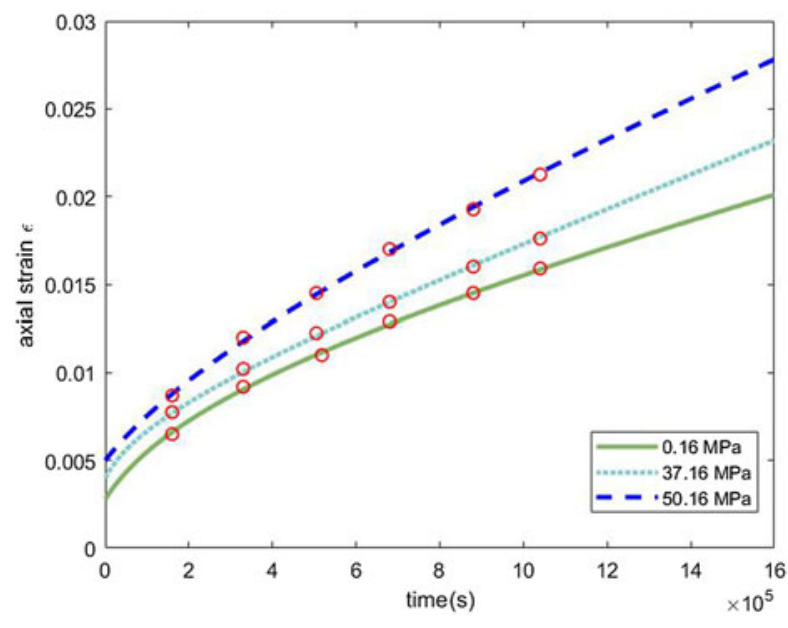

Fig. 5. Axial strain vs. time at different confining pressures, computed with model (8) and the coefficients of Table $1 \mathrm{~b}$. Experimental data (small circles) of Mctigue and others (1985).

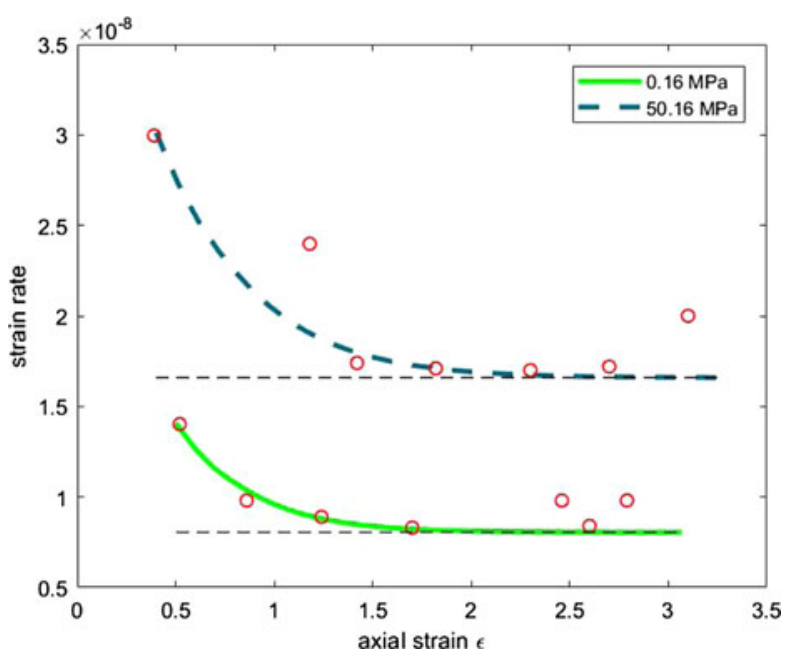

Fig. 6. Strain rate vs. axial strain at different confining pressures, computed with model (8) and the coefficients of Table $2 \mathrm{~b}$. Experimental data (small circles) of Jones and Chew (1983).

(1983) clearly highlight that hydrostatic pressure causes polycrystalline ice to modify its rheology. This type of behavior is comparable to that found in many other materials where the viscosity can vary by a factor of as much as $10^{10 \%}$ (Prusa and others (2012); Bair (2015)). Experimental data concerning glaciers and rock glaciers show appreciable variation of the viscosity with the depth, as shown in Figure 2 of Kannan and Rajagopal (2013).

Tests performed on polycrystalline ice at constant temperature show that ice exhibits characteristics which cannot be adequately described by the classical Newtonian model. Indeed, the ice flow is often represented by a power-law constitutive model (Glen's law) that provides

Table 3. Constitutive coefficients and pressures for creep tests, obtained by fitting to the experimental data of Barrette and Jordaan (2003)

(a) is for the constitutive model (6) (see also Fig. 7)

\begin{tabular}{|c|c|c|c|c|}
\hline$\mu_{0}$ & Pas & & $2.2 \times 10^{19}$ & \\
\hline$k_{u}$ & & & $7.3 \times 10^{-2}$ & \\
\hline$\alpha_{2,0}$ & $\mathrm{Pas}^{2}$ & & $25 \times 10^{21}$ & \\
\hline$k_{\alpha}$ & & & 0.5 & \\
\hline$\alpha_{1}$ & $\mathrm{Pas}^{2}$ & & $-0.65 \times 10^{19}$ & \\
\hline$f_{L}$ & $\mathrm{MPa}$ & -70 & -35 & -15 \\
\hline$f_{A}$ & $\mathrm{MPa}$ & -85 & -50 & -30 \\
\hline$\epsilon_{0}$ & & $-3.3 \times 10^{-3}$ & $-4 \times 10^{-3}$ & $-2 \times 10^{-3}$ \\
\hline$\beta_{0}$ & & $1-3.3 \times 10^{-3}$ & $1-4 \times 10^{-3}$ & $1-2 \times 10^{-3}$ \\
\hline$a_{0}$ & $s^{-1}$ & $-1.6 \times 10^{-2}$ & $-7.5 \times 10^{-3}$ & $-5.6 \times 10^{-3}$ \\
\hline$p$ & $\mathrm{MPa}$ & 75 & 40 & 20 \\
\hline
\end{tabular}

(b) is for the constitutive model (8) (see also Fig. 8

\begin{tabular}{|c|c|c|c|c|}
\hline $\begin{array}{l}\mu_{0} \\
k_{u}\end{array}$ & Pas & & $\begin{array}{c}1.8 \times 10^{18} \\
4 \times 10^{-1}\end{array}$ & \\
\hline$\alpha_{2,0}$ & $\mathrm{Pas}^{2}$ & & $1.85 \times 10^{23}$ & \\
\hline$k_{\alpha}$ & & & 1.1 & \\
\hline$\alpha_{1}$ & $\mathrm{Pas}^{2}$ & & $-4.3 \times 10^{19}$ & \\
\hline$f_{L}$ & $\mathrm{MPa}$ & -70 & -35 & -15 \\
\hline$f_{A}$ & $\mathrm{MPa}$ & -85 & -50 & -35 \\
\hline$\epsilon_{0}$ & & $-3.3 \times 10^{-3}$ & $-4 \times 10^{-3}$ & $-2 \times 10^{-3}$ \\
\hline$\beta_{0}$ & & $1-3.3 \times 10^{-3}$ & $1-4 \times 10^{-3}$ & $1-2 \times 10^{-3}$ \\
\hline$a_{0}$ & $\mathrm{~s}^{-1}$ & $-1.6 \times 10^{-2}$ & $-7.5 \times 10^{-3}$ & $-5.6 \times 10^{-3}$ \\
\hline$p$ & $\mathrm{MPa}$ & 75 & 40 & 20 \\
\hline
\end{tabular}




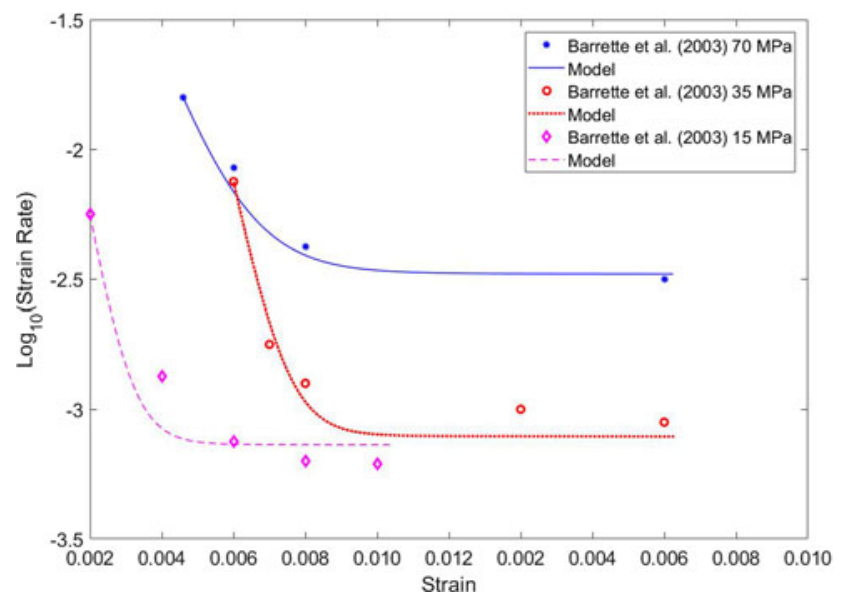

Fig. 7. Strain rate vs. axial strain at different confining pressures, computed with model (6) and the coefficients of Table $3 a$. Experimental data (small circles) of Barrette and Jordaan (2003).

good estimates of shear stresses and overall kinematics in simple flow configurations. However, such a model excludes any normal stress effect, which is of great significance in ice mechanics. For this reason, here we consider a second-order fluid model that provides excellent agreement with primary and secondary creep. In particular, we generalize the model of Mctigue and others (1985) and that of Man and Sun (1987), by proposing the constitutive Eqns (6) and (8), whose rheological parameters depend on the pressure $p$. Model (8) accounts for both shear thinning (as for Glen's law) and normal stress differences.

To assess the suitability of the proposed model, we determine the rheological coefficients using the experimental data referring to primary and secondary creep presented by Jones and Chew (1983), Mctigue and others (1985) and Barrette and Jordaan (2003). The agreement is satisfactory, showing that both models are well suited for capturing the behavior of the strain curves as the pressure increases as proposed by Mctigue and others (1985), the two strain rate vs. strain curves of Fig. 1 in Jones and Chew (1983) and the three strain rates vs. strain curves of Fig. 3 in Barrette and Jordaan (2003). One of the quintessential feature of this paper is to show that rheological models with pressure-

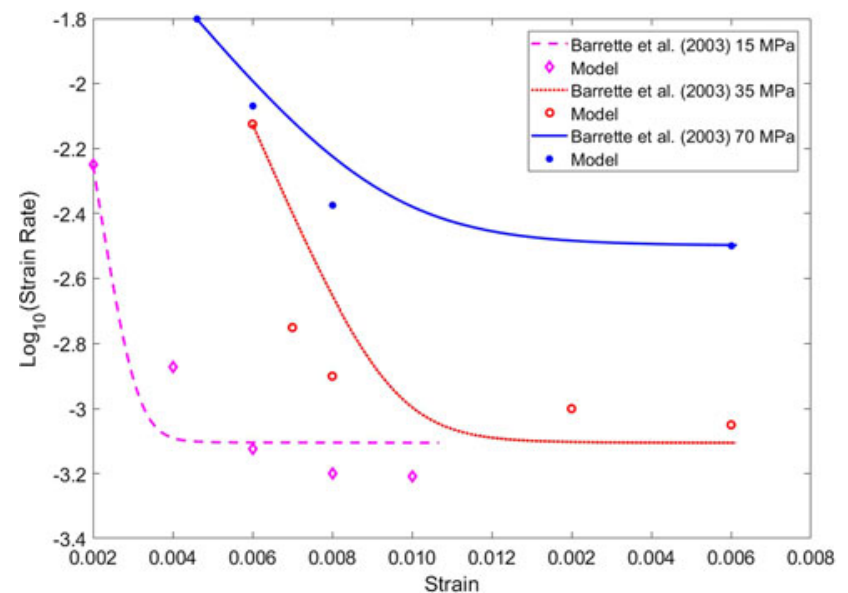

Fig. 8. Strain rate vs. axial strain at different confining pressures, computed with model (8) and the coefficients of Table $3 \mathrm{~b}$. Experimental data (small circles) of Barrette and Jordaan (2003). dependent parameters are able to capture the ice creep enhancing due to high pressure. Actually, Kannan and Rajagopal (2013), using a constitutive model that takes into account the effect of the shear rate, pressure and the volume fraction of the rocks and sand grains trapped within the interstices are also able to reproduce quite satisfactorily the peculiar flow data of the Murt él-Corvatsch glacier.

Concerning the pressure dependence of the rheological parameters of models (6) and (8), we assumed a Barus-type law (9), (10).

Models (6) and (8) are still tentative. Theoretical and experimental work remains to be done in order to discern which of the two models is more appropriate for the creep flow of ice. Indeed, our analysis refers essentially to 1-D axial tests and not to shear tests. The latter could help in the selection of the constitutive model but could also bring out other phenomena such as a pressure-dependent shear thinning effects. Finally, it is well known that temperature greatly influences the flow properties of ice. By taking into account the effects of temperature one may be able to capture the motion more accurately.

\section{ACKNOWLEDGMENTS}

We thank Dr. Ralf Greve, Hokkaido University, for his precious comments and useful suggestions that helped us improve this paper. We express our sincere gratitude to Prof. Raymond Ogden, University of Glasgow, and to Prof. Michel Destrade, NUI Galway, for their various comments on this work. We also thank the referees for their useful suggestions.

\section{REFERENCES}

Bair S (2015) The temperature and pressure dependence of viscosity and volume for two reference liquids. Lubrication Sci. (doi: 10.1002/ls.1302)

Barrette PD and Jordaan IJ (2003) Pressure-temperature effects on the compressive behavior of laboratory-grown and iceberg ice. Cold. Reg. Sci. Technol., 36, 25-36.

Barus C (1893) Isothermals, isopiestics and isometrics relative to viscosity. Am. J. Sci., 45, 87-96.

Bird RB, Amstrong RC and Hassager O (1977) Dynamics of polymeric liquids fluid mechanics, $\mathbf{1}$. Wiley

Bridgman PW (1958) The physics of high pressure. Bell and Sons Ltd, London.

Dunn JE and Fosdick RL (1974) Thermodynamics, stability, and boundedness of fluids of complexity 2 and fluids of second grade. Arch. Ration. Mech. Anal., 56, 191-252.

Dunn JE and Rajagopal KR (1995) Fluids of differential type: critical review and thermodynamic analysis. Int. J. Eng. Sci., 33, 689-729.

Fosdick RL and Rajagopal KR (1979) Anomalous features in the model of 'second order fluids'. Arch. Ration. Mech. Anal., 70, 145-152.

Glen J, W. (1955) The creep of polycrystalline ice. Proc. R. Soc. Lond. A, 228, 519538 (doi: 10.1098/rspa.1955.0066)

Greve R, Zwinger T and Gong Y (2014) On the pressure dependence of the rate factor in Glen's flow law. J. Glaciol., 60, 397-398 (doi: 10.3189/2014JoG14J019)

Jones SJ and Chew HAM (1983) Creep of ice as a function of Hydrostatic Pressure. J. Chem. Phys., 87, 4064-4066 (doi: 10.1021/j100244a013)

Jones Parry E and Tabor D (1973) Effect of hydrostatic pressure on the mechanical properties of polymers: a brief review of published data. J. Mater. Sci., 8, 1510-1516. 
Kannan K and Rajagopal KR (2013) A model for the flow of rock glaciers. Int. J. Non. Linear. Mech., 48, 59-64 (doi: 10.1016/j. ijnonlinmec.2012.06.00)

Kjartanson BH, Shields DH, Domaschuk L and Man CS (1988) The creep of ice measured with the pressuremeter. Can. Geotech. J., 25, 250-261 (doi: 10.1139/t88-029)

Man CS and Sun QX (1987) On the significance of normal stress effects in the flow of glaciers. J. Glaciol., 33, 268-273.

Mctigue DF, Passman SJ and Jones SJ (1985) Normal stress effects in the creep of ice. J. Glaciol., 31, 120-126 (doi: 10.1017/ S0022143000006353)

Passman SL (1982) Creep of a Second Order Fluid. J. Rheol., 26, 373-385 (doi: 10.1122/1.549681)

Prusa V, Srinivasan S and Rajagopal KR (2012) Role of pressure dependent viscosity in measurements with falling cylinder viscometer. Int. J. Non. Linear. Mech., 47, 743-750 (doi: 10.1016/ j.ijnonlinmec. 2012.02.001)

Rajagopal KR (1995) On boundary conditions for fluids of the differential type. In Sequeira A ed. Navier-Stokes equations and related nonlinear problems. Plenum Press, New York.

Rajagopal KR (2003) Implicit constitutive relations. Appl. Math., 28, 279-319.

Rajagopal KR (2006) On implicit constitutive theories for fluids. J. Fluid. Mech., 550, 243-249 (doi: 10.1017/S0022112005 008025)

Rajagopal KR (2015) Remark on the notion of 'pressure'. Int. J. Non. Linear. Mech., 71, 165-172 (doi: 10.1016/j.ijnonlinmec.2014. 11.031)

Rajagopal KR and Kaloni PN (1989) Some remarks on boundary conditions for fluids of the differential type. In Graham GAC and Malik SK, eds. Continuum Mechanics and Its Applications. Hemisphere, New York
Rajagopal KR and Saccomandi G (2016) A Novel approach to the description of constitutive relations. Front. Mater., 3, 335-346.

Rajagopal KR and Szeri AZ (2003) On an inconsistency in the derivation of the equations of elastohydrodynamic lubrication. Proc. Roy Soc. Lond. Ser. A. Math. Phys. Eng. Sci., 459, 2771-2786 (doi: 10.1098/rspa)

Rajagopal KR, Saccomandi G and Vergori L (2012) Flow of fluids with pressure- and shear-dependent viscosity down an inclined plane. J. Fluid Mech., 706, 173-189.

Rajagopal KR, Saccomandi G and Vergori L (2015) On the approximation of isochoric motions of fluids under different flow conditions. Proc. Roy. Soc. London Ser. A, 471, 2150-2159.

Rigsby GP (1958) Effects of hydrostatic pressure on velocity of shear deformation of single ice crystals. J. Glaciol., 3, 271-278 (doi: 10.1017/S0022143000023911)

Sahaphol T and Miura S (2005) Shear moduli of volcanic soils. Soil Dyn. Earthq. Eng., 25, 157-165.

Schulson EM and Duval P (2009) Creep and frecture of ice. Cambridge University Press

Shin ES and Pae RD (1992) Effects of hydrostatic pressure on the torsional shear behavior of graphite/epoxy composites. J. Compos. Mater., 26, 462-485.

Szeri AZ (2005) Fluid film lubrication: theory and design. Cambridge University Press

Truesdell C and Noll W (1992) The non-linear field theories of mechanics. Springer-Verlag

Truesdell C and Rajagopal KR (2000) An introduction to the Mechanics of Fluids. Birkhaüser, Boston

Van Der Veen CJ (2013) Fundamentals of glacier dynamics, 2nd edn. CRC Press, Boca Raton

Weertman J (1983) Creep deformation of ice. Annu. Rev. Earth. Planet. Sci., 11, 215-240 (doi: 10.1146/annurev.ea.11.050183.001243)

MS received 28 March 2018 and accepted in revised form 25 April 2019; first published online 15 July 2019 